\title{
Competencias laborales del auditor de servicios de salud: un análisis funcional Venezuela, 2008
} A functional analysis of healthcare auditors' skills in Venezuela, 2008

\author{
Mónica S. Chirinos-Muñoz \\ Universidad del Zulia. Programa de Administración del Sector Salud. Maracaibo, Venezuela. \\ monicasuchm@yahoo.com
}

Recibido 13 Julio 2010/Enviado para Modificación 20 Diciembre 2010/Aceptado 28 Diciembre 2010

\section{RESUMEN}

Objetivo Identificar las competencias laborales, específicas, genéricas, básicas y los valores que debe poseer el auditor de servicios de salud, a través de un análisis funcional.

Métodos Implementación de la técnica del análisis funcional con 10 expertos, identificando competencias específicas, básicas, genéricas y valores, mediante un proceso de análisis lógico deductivo.

Resultados Se obtiene el mapa funcional que inicia con el establecimiento de un propósito clave basado en la mejora de la calidad de la atención y servicios de salud; de donde surgen las tres funciones claves, y luego se inicia un proceso de desglose continúo desde las funciones principales, unidades de competencia hasta los elementos de competencia que definen lo que el auditor de servicios de salud es capaz de hacer.

Discusión Este mapa funcional refleja de forma detallada las funciones simples y complejas que un auditor de servicios de salud según la metodología del análisis funcional debería aplicar en el campo laboral, atendiendo el enfoque gerencial con visión de mejora de la calidad de la atención y servicios de salud. Esta metodología que expresa un proceso de socialización lógico-deductivo provee de una información consensuada por expertos que validan cada uno los elementos de competencia global.

Palabras Clave: Competencias, laboral, auditoría (fuente: DeCS,BIREME).

\section{ABSTRACT}

Objectives Using functional analysis for identifying the basic, working, specific and generic skills and values which a health service auditor must have.

Methods Implementing the functional analysis technique with 10 experts, identifying specific, basic, generic skills and values by means of deductive logic.

Results A functional map was obtained which started by establishing a key purpose based on improving healthcare and service quality from which three key functions emerged. The main functions and skills' units were then broken down into the competitive elements defining what a health service auditor is able to do. 
Discussion This functional map (following functional analysis methodology) shows in detail the simple and complex tasks which a healthcare auditor should apply in the workplace, adopting a forward management approach for improving healthcare and health service quality. This methodology, expressing logical-deductive awareness raising, provides expert consensual information validating each element regarding overall skills.

Key Words: Skills, work, auditing (source: $M e S H, N L M$ ).

$\mathrm{E}$ l avance técnico en materia de recursos humanos en salud; ha evidenciado claramente los obsoletos y erróneos modelos gerenciales que actualmente se aplican; trayendo esto como consecuencia la disminución en la prestación de servicios productivos, eficientes, y de calidad. En este sentido, la problemática germina en la gestión del recurso humano, que muchas veces asume competencias que no son pertinentes en el campo laboral.

Por lo anteriormente expuesto, como objetivo general se identificaron las competencias del auditor en servicios de salud, por medio del método de análisis funcional (1), establecido por la Organización Internacional del Trabajo, como una metodología que incorpora innovaciones en el sector salud y educación.

Igual vale mencionar las valiosas propuestas que en materia educativa se han establecido y que buscan igual que este articulo engranar de forma más sólida y pertinente los modelos educativos y el campo laboral; para así garantizar profesionales proactivos y capaces de responder a las demandas volubles e innovadoras del sector.

Por último, con esta investigación se busca ofrecer un aporte importante por su contenido de valor tecnológico y funcional ya que dará apertura a otras propuestas laborales y aporta al nivel educativo los pilares básicos de la formación basada en competencias.

\section{MÉTODOS}

Se aplicó la metodología de análisis funcional, según los parámetros establecida por la misma (2), en los siguientes pasos:

- Selección de diez "expertos", con experiencia y competencias demostradas en el área de auditoría, tanto en salud como en el área administrativa. 
- Reunión con el grupo seleccionado en un espacio físico, previamente acondicionado para la actividad. Inició con un taller introductorio dirigido por un experto en análisis funcional, sobre el enfoque de competencias, utilidad, características, propósitos, metodología general con entrega de material didáctico que recopilaba ideas y conocimientos sobre competencias, y principios generales de la auditoria en salud (2-9) ya que el enfoque debe ser adaptado a las necesidades del sector de las instituciones.

- Construcción del mapa funcional (2), por medio de una lluvia de ideas y (10) bajo un espacio de análisis, reflexión, discusión, construcción y socialización, definiendo el propósito clave.

- Inicio del proceso de desagregación progresiva, en el que el panel de expertos discutía y realizaba aportes individuales sobre las funciones que correspondían a una lógica deductiva que avanza desde lo general a lo particular, bajo el enfoque de causa - consecuencia que contribuye al logro del propósito clave y respondiendo a la interrogante: ¿Qué hay que hacer para que esto se logre?

- Luego de llevar el proceso de desagregación a su máxima expresión, se detuvo el proceso ya que correspondía a la identificación de un elemento de competencia. A este nivel se enlaza la frase: "La persona debe ser capaz de...".

Con este paso se dio fin al taller en el que se aplicó la metodología del análisis funcional. Posteriormente se realizó el análisis de los datos obtenidos y se organizaron como competencias.

\section{RESULTADOS}

El mapa funcional expresado a continuación, hace la representación del proceso de desagregación que se planteó. En primer término, se obtuvo el propósito principal del Auditor en servicios de salud; el cual responde a la finalidad primaria que cumple la figura del auditor integral en una institución. Consecutivamente, orientados con el enunciado se obtienen tres funciones claves, de las cuales se genera toda la información operativa de la labor del auditor institucional, luego transcurre por las funciones principales, unidades de competencia y elementos de competencia como último planteamiento de la metodología. 
Figura 1. Mapa funcional

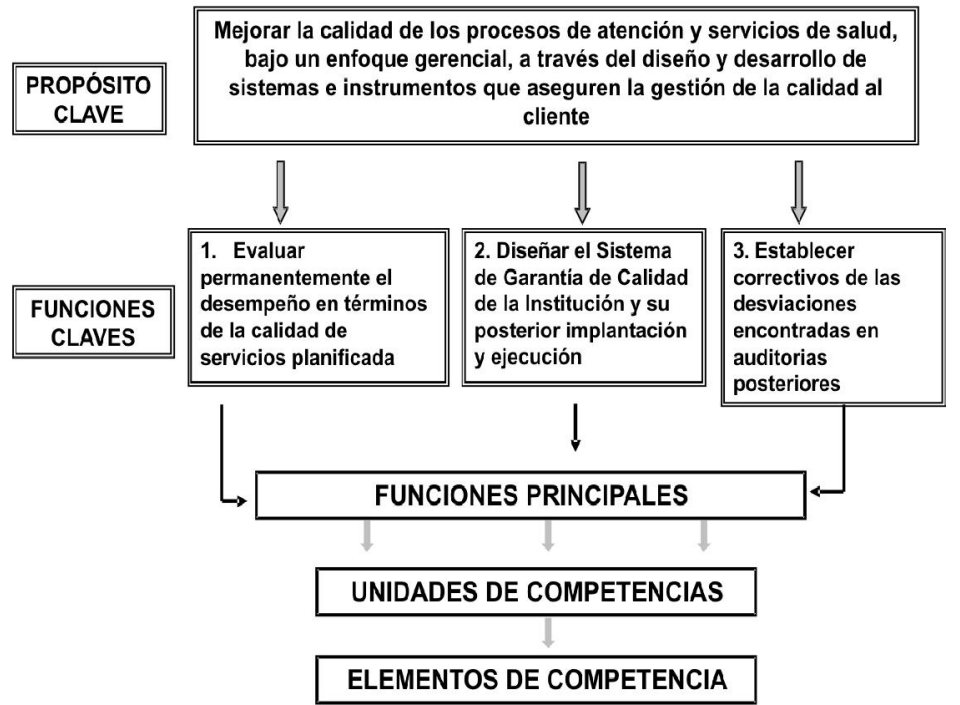

Cuadro 1. Función clave 1 del auditor de servicios de salud Análisis funcional

\begin{tabular}{|c|c|c|c|}
\hline $\begin{array}{c}\text { Funciones } \\
\text { Claves }\end{array}$ & $\begin{array}{l}\text { Funciones } \\
\text { Principales }\end{array}$ & $\begin{array}{l}\text { Unidades de } \\
\text { Competencia }\end{array}$ & Elementos de Competencia \\
\hline $\begin{array}{l}\text { 1. Evaluar } \\
\text { permanente } \\
\text { mente el } \\
\text { desempeño } \\
\text { en términos } \\
\text { de la calidad } \\
\text { de servicios } \\
\text { planificada. }\end{array}$ & $\begin{array}{l}\text { Realiza } \\
\text { mediciones } \\
\text { para valorar } \\
\text { la calidad } \\
\text { del servicio. }\end{array}$ & $\begin{array}{l}\text { Diseñar } \\
\text { estándares de } \\
\text { calidad. }\end{array}$ & $\begin{array}{l}\text { a. Identificar elementos de } \\
\text { estructura, procesos y resultados. } \\
\text { b. Conoce Requisitos legales y } \\
\text { FODA } \\
\text { c. Analizar el comportamiento del } \\
\text { indicador a través del tiempo } \\
\text { d. Conocer los estándares } \\
\text { internacionales. } \\
\text { e. Monitorear la referencia de } \\
\text { indicadores por elementos } \\
\text { teóricos. } \\
\text { f. Coordinar el proceso de } \\
\text { valoración por consenso de los } \\
\text { indicadores. } \\
\text { g. Clarificar responsabilidad } \\
\text { funcional vs comportamiento del } \\
\text { indicador y su desviación según } \\
\text { referencia. } \\
\text { h. Identificar las necesidades } \\
\text { reales de los usuarios. } \\
\text { i. Diseñar las guías de manejo } \\
\text { (Medicina Basada en Evidencia), } \\
\text { supervisando la construcción de su } \\
\text { contenido. }\end{array}$ \\
\hline
\end{tabular}


Cuadro 2. Función clave 2 del auditor de servicios de salud Análisis funcional

\begin{tabular}{|c|c|c|c|}
\hline Funciones claves & Funciones principales & Unidades de competencia & Elementos de competencia \\
\hline \multirow[t]{8}{*}{$\begin{array}{l}\text { 2. Diseño del Sistema de } \\
\text { garantía de Calidad de la } \\
\text { institución de salud, para su } \\
\text { posterior implantación y } \\
\text { ejecución. }\end{array}$} & \multirow[t]{3}{*}{$\begin{array}{l}\text { Organización de la función de } \\
\text { Garantía de la Calidad. }\end{array}$} & $\begin{array}{l}\text { Organizar Comités de Auditoria en } \\
\text { las Instituciones de salud. }\end{array}$ & $\begin{array}{l}\text { a. Seleccionar profesionales según } \\
\text { experiencia y principios morales y } \\
\text { profesionales demostrados. } \\
\text { b. Definir las competencias y } \\
\text { funciones del comité dentro de la } \\
\text { institución de salud. }\end{array}$ \\
\hline & & $\begin{array}{l}\text { Elaborar un plan de desarrollo de } \\
\text { la función de garantía de la } \\
\text { calidad. }\end{array}$ & $\begin{array}{l}\text { a. Definir la Misión y alcance de las } \\
\text { funciones del SGC. } \\
\text { b. Elaborar los manuales del SGC y } \\
\text { expedición a todos los servicios. } \\
\text { c. Evaluar las normativas sobre el } \\
\text { cumplimiento de requisitos minimos } \\
\text { de las entidades prestadoras de } \\
\text { salud y autorregulaciones: colegios } \\
\text { de médicos, enfermeria, etc. }\end{array}$ \\
\hline & & $\begin{array}{l}\text { Gestionar la asignación de } \\
\text { infraestructura física, recursos } \\
\text { materiales y humanos. }\end{array}$ & $\begin{array}{l}\text { a. Elaborar lista de necesidades y } \\
\text { propuesta justificando la estructura } \\
\text { fisica ye insumos para la } \\
\text { implantación del } \$ \mathrm{GC} \text {. }\end{array}$ \\
\hline & \multirow[t]{2}{*}{$\begin{array}{l}\text { Planificación de la } \\
\text { documentación y formalización } \\
\text { de procesos y procedimientos. }\end{array}$} & $\begin{array}{l}\text { Identificar los procesos de la } \\
\text { institución. }\end{array}$ & $\begin{array}{l}\text { a. Observar la secuencia e } \\
\text { interacción de los procesos en los } \\
\text { servicios y la institución en general. } \\
\text { b. Verificar políticas generales de la } \\
\text { institución, organigramas, etc. } \\
\text { c. Elaborar insstrumentos } \\
\text { (cuestionarios, buzón de quejas, } \\
\text { etc.). }\end{array}$ \\
\hline & & $\begin{array}{l}\text { Sistematizar la información } \\
\text { proveniente de los servicios, etc. }\end{array}$ & $\begin{array}{l}\text { a. Recolectar, organizar y tabular la } \\
\text { información según variables } \\
\text { internas y externas. }\end{array}$ \\
\hline & \multirow[t]{3}{*}{$\begin{array}{l}\text { Diseñar mecanismos de } \\
\text { gestión y mejoramiento } \\
\text { continuo de la calidad. }\end{array}$} & $\begin{array}{l}\text { Identificar necesidades y } \\
\text { demandas de la institución. }\end{array}$ & $\begin{array}{l}\text { a. Supervisar continuamente los } \\
\text { procesos (directa e indirectamente) } \\
\text { b. Evaluar los indicadores de } \\
\text { rendimiento y gestión hospitalaria y } \\
\text { de salud. } \\
\text { c. Aplicación de instrumentos de } \\
\text { estudio y análisis de desempeño o } \\
\text { d. Aplicar mecanismos e } \\
\text { instrumentos para conocer el nivel } \\
\text { de satisfacción de los usuarios, } \\
\text { e. Evaluar reclamos, sugerencias, etc. }\end{array}$ \\
\hline & & $\begin{array}{l}\text { Evaluar necesidades y } \\
\text { expectativas personales } \\
\text { del recurso humano }\end{array}$ & $\begin{array}{l}\text { a. Atender las necesidades } \\
\text { propuestas por el personal. } \\
\text { b. Verificar la viabilidad de las } \\
\text { propuestas. } \\
\text { c. Aplicación de técnicas para } \\
\text { identificar ideas de mejoramiento } \\
\text { del área de trabajo o desempeño. }\end{array}$ \\
\hline & & $\begin{array}{l}\text { Investigar las ofertas del } \\
\text { entorno cientifico, social, } \\
\text { económico, y cultural para } \\
\text { garantizar la competitividad } \\
\text { en el mercado y la } \\
\text { actualización permanente. }\end{array}$ & $\begin{array}{l}\text { a. Contactar Sociedades cientificas } \\
\text { y gremios que ofrezcan } \\
\text { actividades de capacitación y } \\
\text { actualización. }\end{array}$ \\
\hline
\end{tabular}


Cuadro 3. Función clave 3 del auditor de servicios de salud Análisis funcional

\begin{tabular}{|c|c|c|c|}
\hline Funciones claves & $\begin{array}{l}\text { Funciones } \\
\text { principales }\end{array}$ & $\begin{array}{l}\text { Unidades de } \\
\text { competencia }\end{array}$ & $\begin{array}{c}\text { Elementos de } \\
\text { competencia }\end{array}$ \\
\hline \multirow[t]{2}{*}{$\begin{array}{l}\text { 3. Diseñar un } \\
\text { programa de } \\
\text { Auditoria de } \\
\text { seguimiento }\end{array}$} & $\begin{array}{l}\text { Verificar que las } \\
\text { acciones planteadas } \\
\text { como resultados de } \\
\text { las observaciones se } \\
\text { lleven a la práctica. }\end{array}$ & $\begin{array}{l}\text { Comunicar los } \\
\text { resultados de las } \\
\text { observaciones y las } \\
\text { acciones planteadas a } \\
\text { los que realizan los } \\
\text { procesos y el director } \\
\text { de la institución. }\end{array}$ & $\begin{array}{l}\text { - Elaboración de } \\
\text { informes de recopilaron } \\
\text { de información: } \\
\text { desviaciones y } \\
\text { correctivos sugeridos. } \\
\text { - Atender las } \\
\text { recomendaciones de } \\
\text { los protagonistas que } \\
\text { elaboran los procesos. }\end{array}$ \\
\hline & $\begin{array}{l}\text { Constatar las } \\
\text { acciones que se } \\
\text { llevaron a cabo para } \\
\text { instrumentar las } \\
\text { recomendaciones. }\end{array}$ & $\begin{array}{l}\text { Aplicar instrumentos } \\
\text { de verificación a los } \\
\text { procesos o elementos } \\
\text { susceptibles. }\end{array}$ & $\begin{array}{l}\text { - Elaborar lista de } \\
\text { procesos que ameriten } \\
\text { corrección y } \\
\text { seguimiento. } \\
\text { - Elaborar instrumentos } \\
\text { para verificar } \\
\text { sistemáticamente } \\
\text { desviaciones } \\
\text { encontradas vs } \\
\text { correcciones } \\
\text { planteadas. } \\
\text { - Diseñar herramientas } \\
\text { para identificación de } \\
\text { soluciones. } \\
\text { - Aplicar instrumentos } \\
\text { de verificación } \\
\text { sistemática de } \\
\text { desviaciones vs } \\
\text { correctivos aplicados. } \\
\text { - Redactar informes } \\
\text { comparativos de } \\
\text { procesos evaluados, } \\
\text { desviaciones y } \\
\text { correctivos aplicados. }\end{array}$ \\
\hline
\end{tabular}

Cuadro 4. Competencias básicas del auditor de servicios de salud Análisis funcional

COMPETENCIAS BASICAS

-Dominio de normativas y aspectos legales que rigen el sector salud y los procesos.

-Dominio de Conocimientos en métodos de trabajo y técnicas de estudio, redacción y síntesis

-Dominios de programas de informática y de computación en general.

-Dominio de tareas y procesos profesionales asistenciales, administrativos (general y

especializado.

-Manejo y destreza en la aplicación de la metodología cientifica en salud que apoye el análisis y la consistencia en la información.

-Dominio del o los idiomas que sean parte de la dinámica de trabajo de la organización -Dominio de técnicas estadistica.

\section{DISCUSIÓN}

La técnica del Análisis Funcional es una herramienta que facilita la identificación de competencias de tal forma, que estas se visualicen de manera puntual, objetiva, y pertinente, ya que se deriva de un proceso de desagregación progresiva, basándose en la experiencia de quienes intervienen en el proceso asegurando la adaptación al contexto (2-11). 
Cuadro 5. Competencias genéricas del auditor de servicios de salud. Análisis funcional

\begin{tabular}{|c|c|}
\hline Competencias genéricas & $\begin{array}{c}\text { Descripción de la conducta/indicadores de } \\
\text { desempeño }\end{array}$ \\
\hline Apertura al cambio & $\begin{array}{l}\text { Poseer conceptos amplios y flexibles para } \\
\text { interpretar la realidad cambiante de las } \\
\text { instituciones, (procesos, políticas, la dinámica } \\
\text { de las profesiones de la salud y de los } \\
\text { conocimientos científicos). }\end{array}$ \\
\hline $\begin{array}{l}\text { Expresión clara de ideas y excelente } \\
\text { comunicación }\end{array}$ & $\begin{array}{l}\text { Capacidad para hablar en público, y con } \\
\text { expresión verbal fluida sin desequilibrios } \\
\text { emocionales o falta de madurez, y } \\
\text { comunicación asertiva. }\end{array}$ \\
\hline Sentido Institucional & $\begin{array}{l}\text { Respeta y obedece a la institución a la que } \\
\text { pertenece, defiende siempre la autaridad que } \\
\text { los asiste y la confianza depositada por el } \\
\text { bien común }\end{array}$ \\
\hline Criterio Profesional & $\begin{array}{l}\text { Emplea su capacidad de discernimienta de } \\
\text { forma equilibrada, para contribuir a ejercer } \\
\text { con eficiencia, responsabilidad, y madurez su } \\
\text { función que le permita comparar la norma y } \\
\text { sus desviaciones dentro de un contexto } \\
\text { institucional real para emitir juicios sólidos, } \\
\text { consistentes y específicos del área }\end{array}$ \\
\hline Actitud de supervisión & $\begin{array}{l}\text { Vigila el estricto cumplimiento y apego a las } \\
\text { disposiciones jurídicas y administrativas, con } \\
\text { el propósito de que sus informes sirvan de } \\
\text { orientación en la toma de decisiones. }\end{array}$ \\
\hline Objetividad & $\begin{array}{l}\text { Evita juicios que no respondan estrictamente } \\
\text { al propósito de las acciones de vigilancia, y } \\
\text { cuida que las recomendaciones derivadas del } \\
\text { ejercicio de su tarea no presenten omisiones } \\
\text { o fallas que puedan parecer premeditadas por } \\
\text { falta de cuidado. No se involucra en forma } \\
\text { personal en los hechos, al margen de sus } \\
\text { preferencias personales. }\end{array}$ \\
\hline Creatividad & $\begin{array}{l}\text { Atiende con solvencia, entereza y dedicación, } \\
\text { sus labores, buscando siempre lograr } \\
\text { resultados óptimos con los recursos } \\
\text { disponibles. Es positivo e innovador en el } \\
\text { desarrollo de su trabajo }\end{array}$ \\
\hline Apertura al Cambio & $\begin{array}{l}\text { Poseer conceptos amplios y flexibles para } \\
\text { interpretar la realidad cambiante de las } \\
\text { instituciones, sus procesos, políticas, la } \\
\text { dinámica propia de estas, de las profesiones de } \\
\text { la salud y del conocimiento científico. }\end{array}$ \\
\hline Trabajo en equipo & $\begin{array}{l}\text { Capacidad para apreciar las actitudes y las } \\
\text { aptitudes de quienes guía, para asignar tareas } \\
\text { y valorar las ideas que aportan el resto del } \\
\text { grupo }\end{array}$ \\
\hline
\end{tabular}




\begin{tabular}{cl}
\hline Competencias genéricas & $\begin{array}{c}\text { Descripción de la conducta/indicadores de } \\
\text { desempeño }\end{array}$ \\
\hline Aprendizaje continúo & $\begin{array}{l}\text { Disposición e interés permanente hacia el } \\
\text { desarrollo, adquisición y transferencia de } \\
\text { nuevos conocimientos y tecnologías, } \\
\text { demostrando interés para compartir, guiar, } \\
\text { orientar o entrenar a otros, para construir y } \\
\text { preservar la memoria corporativa de la } \\
\text { organización de salud. }\end{array}$ \\
& $\begin{array}{l}\text { Sus papeles de trabajo deben ser lo mas } \\
\text { objetivo, claro y sinóptico posible para que no } \\
\text { se disperse la idea de lo que se requiere } \\
\text { expresar. }\end{array}$ \\
Capacidad de sintesis & $\begin{array}{l}\text { Es hábil para integrar ideas de diversa } \\
\text { naturaleza de forma asertiva y dirige un grupo } \\
\text { de personas para obtener eficientes resultados. }\end{array}$ \\
Capacidad de Liderazgo & $\begin{array}{c}\text { Promueve y anticipa actitudes a los hechos y } \\
\text { acciones en pro de la institución. }\end{array}$ \\
Pro actividad & $\begin{array}{c}\text { Aplica el Conjunto de normas que ordena y le } \\
\text { imprimen sentido a los valores y principios de } \\
\text { conducta. }\end{array}$ \\
\hline
\end{tabular}

Cuadro 6. Valores del auditor de servicios de salud Análisis funcional

\begin{tabular}{|c|c|}
\hline Valores & Indicadores de conducta \\
\hline Honestidad & $\begin{array}{l}\text { Aceptar su condición y dar su mejor esfuerzo } \\
\text { con sus propios recursos, evitando aceptar } \\
\text { compromisos o tratos externos. }\end{array}$ \\
\hline Credibilidad & $\begin{array}{l}\text { Desempeñarse con integridad de } \\
\text { pensamiento y actos, para que su función } \\
\text { pública sea ejemplo en su gestión. }\end{array}$ \\
\hline Imparcialidad & $\begin{array}{l}\text { No involucrarse en forma personal en los } \\
\text { hechos, conservando su objetividad al } \\
\text { margen de preferencias personales. }\end{array}$ \\
\hline Compromiso & $\begin{array}{l}\text { Apego a las políticas de la institución y a la } \\
\text { responsabilidad que le fue encomendada. }\end{array}$ \\
\hline Responsabilidad & $\begin{array}{l}\text { Observar una conducta profesional, } \\
\text { cumpliendo con sus encargos oportuna } \\
\text { eficientemente. }\end{array}$ \\
\hline Confidencialidad & $\begin{array}{l}\text { Conservar en secreto la información y no } \\
\text { utilizarla en beneficio propio o de intereses } \\
\text { ajenos. }\end{array}$ \\
\hline Disciplina & $\begin{array}{l}\text { Tener presente las obligaciones para consigo } \\
\text { mismo y la organización a la que presta sus } \\
\text { servicios. Apego a las normas. }\end{array}$ \\
\hline Buenas relaciones Humanas & $\begin{array}{l}\text { Debe ser amable en el trato, y no intimidar ni } \\
\text { utilizar el castigo. }\end{array}$ \\
\hline Asertivo & $\begin{array}{l}\text { Buen manejo de las emociones y de las } \\
\text { situaciones que se puedan presentar. }\end{array}$ \\
\hline Equilibrio & $\begin{array}{l}\text { No perder la dimensión de la realidad y el } \\
\text { significado de los hechos. }\end{array}$ \\
\hline Humildad & $\begin{array}{l}\text { Reconocer la actividad en salud como un acto } \\
\text { de servicio. }\end{array}$ \\
\hline Integridad & $\begin{array}{l}\text { Preservar sus valores por encima de las } \\
\text { presiones. }\end{array}$ \\
\hline
\end{tabular}


En este sentido, respondiendo a criterios de pluralidad de pensamiento bajo un enfoque unificado; se evidencia en un primer término que el mapa funcional obtenido hace la representación del proceso planteado con un enfoque funcional, e integral, que responde a las demandas sanitarias en términos técnicos-prácticos. De igual forma, emergen las competencias genéricas, básicas y los valores como complemento útil para la propuesta global de las competencias.

Secundariamente es importante comentar, que el propósito clave derivado tiene como base de competencia, el mejoramiento de la calidad de la atención en salud desde un punto de vista gerencial, que promueve las "oportunidades de mejora" (4) como base de integración y participación de todos los miembros de la institución. Finalmente en un tercer término, se puede comentar que las 3 funciones claves indicadas son el núcleo del desempeño laboral del auditor en salud que sustenta en un futuro la creación pertinente de currículos basados en competencia (11-12).

Por lo expuesto anteriormente se puede resaltar; que la articulación de los componentes propios de una norma de competencia ofrece el inicio visionario y proyectado para la ingeniería curricular basado en competencia laboral en el área de salud, lo cual garantiza la inserción efectiva del egresado con un perfil pertinente, adecuado y adaptado a las demandas del sector. Así mismo, presenta al proceso de gestión de recursos humanos institucional una herramienta de trabajo para actualizar los perfiles de cargo •

\section{REFERENCIAS}

1. Oficina Internacional del Trabajo (CINTERFOR/OIT). Formación Basada en Competencia Laboral. Situación Actual y Perspectivas. Primera Edición. Montevideo. Cinterfor; 1997.

2. Irigoin M, Vargas F. Competencia Laboral: Manual de conceptos, métodos y aplicaciones en el sector salud. Programa de desarrollo de Recursos humanos. Primera Edición. Montevideo: Cinterfor; 2002.

3. Mejía B. Auditoría Médica. Tercera edición. Bogotá, Colombia. ECOE Ediciones; 2000.

4. Malagón L, Galán M, Pontón L. Auditoría en salud. Segunda edición. Bogotá, Colombia. Editorial médica panamericana; 2003.

5. Organización Mundial de la salud. Calidad de la atención: seguridad del paciente. 55 a Asamblea Mundial de la Salud 2002; (WHA 55.18) [Internet] Disponible en: www.paho.org/Spanish/AD/THS/EV/blood-4ta-resolucion.pdf. Consultado enero de 2007.

6. Osorio G, Sayes N, Fernández L, Araya E, Poblete D. Auditoría médica. Herramienta de gestión moderna subvalorada. Rev. Med. Chile. 2002; 130 (2): 226-229. 
7. MINEDUC-DIVESUP. Área de Formación y Capacitación para el Trabajo del Programa de Competencias Laborales Identificación de competencias laborales a través del Análisis Funcional. Primera Edición. Catastro Ediciones. Uruguay. 2003.

8. Reyes L, Sánchez M. Modelo de gerencia académica de calidad para el postgrado de la facultad de medicina de La Universidad del Zulia. Primera Edición. Gerencia en América Latina. Maracaibo - Zulia. 2002. p1 - 4.

9. Serrano C. El enfoque de competencias y su utilización en la planificación educativa. Primera Edición. Venezuela. ECOE Ediciones; 2003.

10. Vargas F. 40 preguntas sobre competencia laboral. Primera Edición. Montevideo. CINTERFOR; 2004.

11. Posner G. Análisis del Currículo. Segunda Edición. Bogotá. Mc Graw Hill; 2002.

12. Pinto C. Currículo por Competencias: necesidad de una nueva escuela. Revista de Educación y Cultura. Lima. 1999; № 43. 